\title{
Total cow's milk protein in cookies: the first interlaboratory comparison with a well-defined measurand fit for food allergen risk assessment
}

\author{
Fernando Cordeiro ${ }^{1}{ }^{10}$. Elena Cubero-Leon ${ }^{1} \cdot$ Jørgen Nørgaard $^{1} \cdot$ Maria José Martinez-Esteso $^{1,2} \cdot$ Marcel Brohée $^{1}$. \\ Andreas Breidbach ${ }^{1} \cdot$ Aneta Cizek-Stroh ${ }^{1} \cdot$ Gavin O$^{\prime}$ Connor $^{1,3} \cdot$ Piotr Robouch $^{1} \cdot$ Hendrik Emons $^{1}$
}

Received: 4 August 2020 / Accepted: 23 April 2021 / Published online: 25 May 2021

(c) The Author(s) 2021

\begin{abstract}
A feasibility interlaboratory comparison (ILC) was organised by the European Commission's (EC) Joint Research Centre (JRC) on the determination of the mass fraction of total cow's milk protein in baked cookies. The ILC was organised to support Commission Regulation (EU) 1169/2011 on the provision of food information to consumers, including the mandatory allergen food labelling and the harmonisation of measurement procedures used for food allergen analysis. An incurred baked cookie was prepared at the JRC and samples were sent to participants for analysis. The laboratories were asked to report their results as "mass fraction of total cow's milk protein in baked cookies". This common well-defined measurand was used for the first time in such an ILC to assess the equivalence of measurement results. Homogeneity and stability of the test material were demonstrated to be adequate. An indicative assigned value was established using the JRC single-laboratory validated method based on liquid chromatography coupled to mass spectrometry (LC-MS), independently from the results reported by the participants. Twenty-three laboratories from the European Network of Food Allergen Detection Laboratories (ENFADL), representing 20 European Union (EU) Member States, reported results. Their laboratory performance was assessed using the percent difference $D_{i}$ score. The participating laboratories applied either enzyme-linked immunosorbent assays (ELISA) or LC-MS. Even though the majority of the laboratories used commercially available ELISA test kits, a significant scatter of all the reported results was observed. This indicates that further harmonisation is required for measurement procedures aiming to determine potentially allergenic constituents in food.
\end{abstract}

Keywords Food allergen analysis $\cdot$ Milk protein $\cdot$ Interlaboratory comparison $\cdot$ ELISA $\cdot$ LC-MS

\section{Introduction}

Regulation (EU) 1169/2011 [1] requires the provision of information on 14 priority allergens when intentionally incorporated in the food. While the current EU legislation does not contain specific provisions related to the possible

Fernando Cordeiro

fernando.cordeiro-raposo@ec.europa.eu

1 Joint Research Centre, European Commission, Retieseweg 111, 2440 Geel, Belgium

2 Departamento de Agroquimica Y Bioquimica, University of Alicante, Carrera de San Vincente del Raspeig s/n, San Vincente del Raspeig, 03690 Alicante, Spain

3 Physikalisch-Technische Bundesanstalt, Bundesallee 100, 38116 Braunschweig, Germany and unintentional presence of these allergens, the General Food Law (Regulation (EC) No 178/2002 [2]) obliges food business operators to implement appropriate risk assessment and risk management procedures to assure that food considered unsafe is not placed on the market. Therefore, many food business operators use a precautionary allergen labelling (PAL) to alert consumers on the inadvertent presence of allergens. In order to avoid the unnecessary use of PAL statements, that may reduce consumer choices, quantitative risk assessments are recommended [3]. These can only be performed on the basis of reliable measurement results. However, a major challenge for allergen analysis consists in achieving reliable and comparable measurement results across different measurement procedures addressing different molecular species or parts thereof.

To facilitate the harmonisation and standardisation of food allergen analysis, a European Network for Food 
Allergen Detection Laboratories (ENFADL) has recently been established. One of the first actions of this network was to evaluate the equivalence of measurement results reported by ENFADL members for total cow's milk protein. This ingredient was selected since it is known to cause most allergies in childhood [4] and it causes most of the allergen-based notifications in the Rapid Alert System for Food and Feed (RASFF) [5]. Therefore, the Joint Research Centre (JRC) of the European Commission (EC) organised a feasibility interlaboratory comparison (ILC) for the determination of the mass fraction of total cow's milk protein in food in support of Commission Regulation 1169/2011 [1].

Unlike commercially available proficiency testing schemes for allergen analysis, which provide a consensus value for each ELISA test kit used and allow participants to report in different units, this first of a kind feasibility ILC defined a common measurand as "mass fraction of total cow's milk protein in a baked cookie", independently from the methods used. This allowed an assessment of the equivalence of measurement results obtained by the different laboratories applying different measurement procedures. Since no adequate certified reference material (CRM) was available, a tailor-made test material was prepared and characterised. The corresponding "indicative" assigned value was established using the reference method developed by Martinez-Esteso et al. [6]. This value did not rely on the results reported by the participants.

This manuscript describes the outcome of this unique ILC round.

\section{Experimental}

\section{Preparation of the ILC test item}

Incurred cookies were prepared at the JRC by mixing fat, sugar and flour. Diluted skim milk powder, sodium bicarbonate, ammonium bicarbonate and salt were added to this mixture resulting in a homogeneous dough. Thirty-gram balls were then prepared, flattened to a thickness of $1.5 \mathrm{~mm}$ and cut into circular cookies. The raw cookies were placed on greaseproof paper and baked in an industrial oven for 14 min at $155^{\circ} \mathrm{C}$. The resulting cookies were ground twice: first in a Retsch mill followed by an ultraturax mixer in liquid nitrogen. Three gram portions of the resulting dry powder were finally vacuum-packed in moisture-proof laminated sachets.

\section{Homogeneity and stability}

Homogeneity and stability studies were performed at the JRC. Two independent commercially available ELISA kits (from the Morinaga Institute of Biological Science, Inc.,
Yokohama-Shi, Japan) were used to measure the mass fractions of casein (CAS) and $\beta$-lactoglobulin (LACB) in the incurred baked cookie powder. The studies were performed after the test items had been packed into the final sachets, but before sending them to the participants.

A sufficient number of sachets (10 out of the 200 produced [7]) were randomly selected for the homogeneity assessment. These samples were analysed in duplicates using two ELISA test kits under repeatability condition using 1.0 $\mathrm{g}$ test portions. The results were evaluated according to ISO 13528:2015 [8]. The contribution from inhomogeneity $\left(u_{\text {hom }}\right)$ to the standard measurement uncertainty of the indicative assigned value $u\left(x_{\mathrm{ILC}}\right)$ was calculated using the SoftCRM software [9]. Since no standard deviation for proficiency assessment was established, the following criterion for adequate homogeneity was set: the standard deviation of the measurement results-obtained under repeatability conditions $\left(s_{w}\right)$ and expressing a random variability within sachets-should be smaller than or equal to the standard deviation for measurements between sachets $\left(s_{b}\right)$. This would imply that the uncertainty contribution due to inhomogeneity could not be distinguished from the random variability. This criterion was fulfilled for the marker proteins monitored (CAS and LACB); hence, the test item was considered to be adequately homogeneous.

Based on previous experience, the selected ELISA test kits were considered to have a fit-for-purpose repeatability. It is assumed that, once adequate homogeneity was demonstrated for the selected milk proteins, identical adequate homogeneity would be obtained using LC-MS for the same proteins.

The assessment of stability was performed by keeping 3 sachets at $4{ }^{\circ} \mathrm{C}$ for 8 weeks to cover the complete period of the ILC from value assignment till the deadline for reporting. Three other sachets were stored at the reference temperature of $-80{ }^{\circ} \mathrm{C}$. At the end of the storage period, all samples were measured under repeatability conditions. The uncertainty contribution due to stability $\left(u_{\mathrm{st}}\right)$ was calculated using SoftCRM [9]. Since the slope of the linear regression curve, relating the measured value over time, could not be distinguished from zero, the test item was considered adequately stable at $4{ }^{\circ} \mathrm{C}$ for the testing period. Hence, the standard measurement uncertainty due to instability was set to zero $\left(u_{\mathrm{st}}=0\right)[8]$.

\section{Value assignment and uncertainty estimation}

The indicative assigned value $\left(x_{\text {ILC }}\right)$ of the mass fraction of total cow's milk protein (TCMP) in the baked cookie was determined by applying the single-laboratory validated LC-MS method described by Martinez-Esteso et al. [6], and providing results traceable to the International System of Units (SI). It is based on the measurement of selected marker 
peptides by mass spectrometry and the conversion of the corresponding MS signals to the not-directly measurable TCMP mass fraction. In brief, proteins were extracted from the cookie test material and an aliquot of the cookie extract was fortified with a solution of stable-isotope-labelled isotopologues of the target peptides. The cookie extract, containing the stable-isotope-labelled isotopologues, was digested and cleaned-up. From the final solution, eleven peptides specific to the five constituent proteins of TCMP were measured by LC-MS: four peptides for $\alpha_{\mathrm{S} 1^{-}}$casein (CASA1), two for $\alpha_{\mathrm{S} 2^{-}}$ casein (CASA2), two for $\beta$-casein (CASB), one for $\kappa$-casein (CASK) and two for $\beta$-lactoglobulin (LACB).

Similarly, calibration blends were prepared from a baked cookie free of TCMP and fortified with a reference solution of the above-mentioned non-labelled peptides. The purities of the non-labelled peptides were determined by amino acid analysis [10]. All aliquots and fortifications were prepared gravimetrically using an analytical calibrated balance and masses were recorded.

According to Gellrich et al. [11], the sum of the individual mass fractions of the five selected marker proteins contributes to $92 \%$ of the total mass fraction of the TCMP. Thus, a compositional factor $\left(f_{s}\right)$ taking into account the part of TCMP, which was not covered by the five marker proteins, was set to $f_{s}=1.087(=1 / 0.92)$. Finally, a value of $11.8 \mathrm{mg} \mathrm{kg}^{-1}$ was obtained by combining measurements for the five proteins into a single result (approach A4 described in [6]). This value was set as the indicative assigned value $\left(x_{\mathrm{ILC}}\right)$.

The associated standard measurement uncertainty of the assigned value, $u\left(x_{\text {ILC }}\right)$, was calculated combining the standard uncertainty due to characterisation (or value assignment), homogeneity and stability $\left(u_{\text {char }}, u_{\text {hom }}\right.$ and $u_{\mathrm{st}}$, respectively) as prescribed by ISO 13528:2015 [8]:

$u\left(x_{\mathrm{ILC}}\right)=\sqrt{u_{\mathrm{char}}^{2}+u_{\mathrm{hom}}^{2}+u_{\mathrm{st}}^{2}}$.

A relative measurement uncertainty associated with the LC-MS measurement ( $u_{\text {charrel }}$ ) of $4 \%$ was estimated by Martinez-Esteso et al. applying the approach outlined above [6] (which includes an uncertainty component associated with the compositional factor $f_{s}$ ). A conservative relative uncertainty contribution due to inhomogeneity $\left(u_{\text {hom,rel }}\right)$ of $2.4 \%$ was derived from the homogeneity study, while $u_{\mathrm{st}}$ was set to zero. Hence, a combined standard uncertainty of the informative assigned value $u\left(x_{\mathrm{ILC}}\right)$ of $0.55 \mathrm{mg} \mathrm{kg}^{-1}(k=1)$ was obtained $\left(=11.8 \times \sqrt{0.04^{2}+0.024^{2}}\right)$.

\section{Results and discussion}

The laboratory performance was expressed in terms of the percent difference $\left(D_{i}\right)$ according to ISO 13528:2015 [8]:
$D_{i}=100\left(x_{i}-x_{\mathrm{ILC}}\right) / x_{\mathrm{ILC}}$,

where $x_{i}$ is the measurement result reported by a participant and $x_{\text {ILC }}$ is the indicative assigned value.

A total of 25 results were reported by 22 ENFADL laboratories. Three laboratories registered twice in order to submit results obtained by different measurement procedures. Laboratory L25 reported a truncated value ("higher than"), while L10 reported a total casein result without converting this value to total milk protein. The vast majority of the reported results (23 out of 25 ) were obtained applying different ELISA test kits from various manufacturers.

Figure 1 displays the graphical representation of the reported results including their associated expanded measurement uncertainties (see error bars) as reported by the participants (some of them did not report their measurement uncertainties). Most of the participants submitted values below the indicative assigned range $\left(11.8 \mathrm{mg} \mathrm{kg}^{-1} \pm 0.6\right.$ $\left.(k=1) \mathrm{mg} \mathrm{kg}^{-1}\right)$. This is further confirmed by the significantly lower consensus range of $7.3 \mathrm{mg} \mathrm{kg}^{-1} \pm 0.8(k=1)$ $\mathrm{mg} \mathrm{kg}^{-1}$ calculated using Algorithm A and applying Eq. 6 of ISO 13528:2015 [8].

A large scatter of results has been observed (with $D_{i}$ ranging from $-71 \%$ to $+38 \%$ ), even when the same ELISA test kits, produced by the same manufacturer targeting total milk, were used (see Fig. 1, filled circles). Also, different standard measurement procedures are prescribed by the respective test kit manufacturer, while the

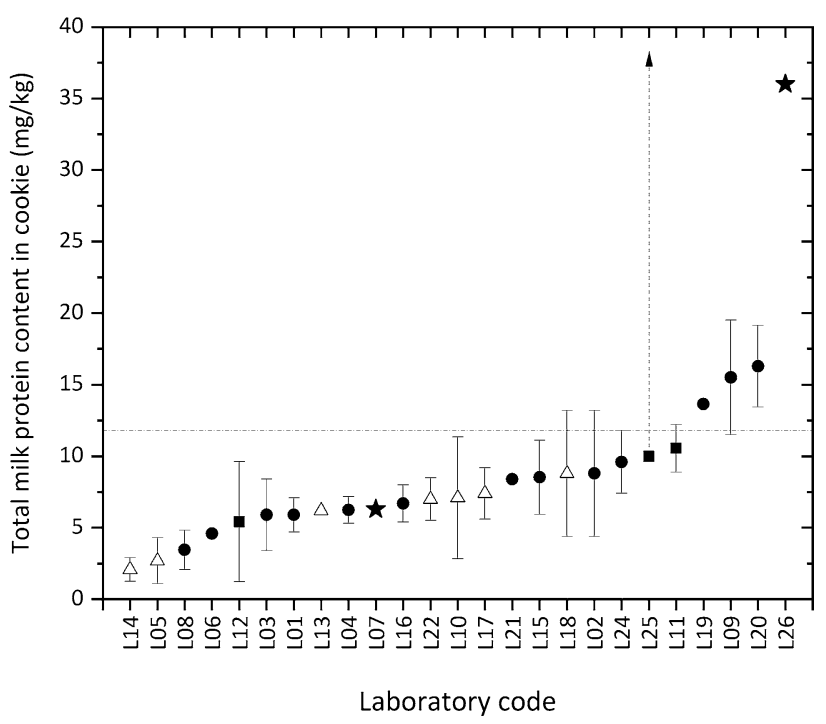

Fig. 1 Measurement results including the associated expanded uncertainties (error bars) as reported by the participants. The following symbols are used: filled circles, for a specific commercial ELISA test kit targeting TCMP; open circles, for other ELISA test kits targeting TCMP; open triangles, for ELISA test kits targeting caseins; stars, for the LC-MS method. L25 reported a "higher than" value. The horizontal line depicts the indicative assigned value 
composition of their calibrants that must be used is seldom disclosed. In addition, specificity and cross-reactivity are poorly documented and should be thoroughly investigated by the laboratories performing the measurement. One may also question the reliability of any conversion factors used (to be applied or imbedded in the calibration curve) to convert the measurement results obtained for the targeted protein into the total cow's milk protein content in the food sample. The low ELISA values reported may be attributed to the use of extraction buffers without reducing agents, which may have hindered the denaturing and release of LACB due to its disulfide bonds. This highlights the importance of understanding the nature of the matrix which has to be analysed and the limitations of each test kit. More information should be provided by the kit manufacturers on the type of extraction buffers used and their suitability for different types of matrices.

Additional experimental information was provided by the participants in a dedicated questionnaire. It included technical questions related to (i) the analytical techniques used by the participants including a separate set of questions regarding the measurement system used by the participant (ELISA, LC-MS), (ii) how the participants ensured the quality of their results (their quality management system) and (iii) their experience analysing these particular targets. Hence, the information collected allowed to identify possible reasons for the diverging results.

In the absence of appropriate certified reference materials (CRM), several laboratories mentioned using the standard reference material NIST 1549a. This SRM is a whole milk powder supplied by the National Institute of Standards and Technology (USA), which provides only an informative (non-certified) protein value. However, this SRM was not intended for allergen detection and may contain "uncommon" ratios or process-related modifications (e.g. lactosylation) of the caseins and whey proteins for a milk product. The processing during the RM production may have affected the total protein and/or marker protein contents as well as their conformation or solubility. Hence, the use of this RM to calibrate or validate a method for the determination of TCMP is deemed questionable.

Two laboratories used LC-MS for the quantification of TCMP (Fig. 1). In order to achieve accurate results by this technique, matrix effects (e.g. ionisation suppression/ enhancement, interferences) must be properly accounted for. In addition, a sufficient equilibration between the matrix, the incurred and the added analytes have to be ensured when applying standard addition. Laboratory L07 applied the standard addition approach using skimmed cow's milk powder, instead of adding stable-isotope-labelled analogues of the analytes. L07 also digested the proteins with trypsin for $60 \mathrm{~min}$ only. This very short duration is likely to result in an incomplete digestion (see Nitride et al. [12] for a detailed description), resulting in the lower value reported.

Laboratory L26 used milk protein in a rice matrix for the calibration of their method and an incurred cookie of $16 \mathrm{mg}$ TCMP $/ \mathrm{kg}$ to convert the measured mass fractions to the reported mass fraction. The protein content of rice is only a fraction of that of wheat and the protein content can vary widely between different wheat varieties. Even though L26 reported the use of six marker peptides for three milk proteins and overnight digestion with trypsin, it is unlikely that the chosen approach is capable to account properly for the matrix effects in the test material. This could explain the highly overestimated reported value.

\section{Conclusions}

In order to ensure accurate measurement results produced by competent laboratories using appropriate measurement procedures for the determination of food allergens in food, this ILC implemented a common well-defined measurand, namely the "mass fraction of total cow's milk protein in baked cookies". A large scatter of reported results was observed even when the same commercially available ELISA test kit was used. This clearly identifies the need for improving the standard operating procedures of the ELISA test kits to ensure the unambiguous interpretation of their applicability. In addition, test kit manufacturers should verify the quality of the calibrants they deliver and should provide a transparent chain for the metrological traceability of the property values carried by the calibrant.

The provision of appropriate CRMs and proficiency testing (PT) schemes, with metrologically traceable assigned values using a validated reference measurement procedure, is essential in improving the accuracy of routine allergen measurements. Currently, the divergent results between different ELISA kit manufacturers and between different measurement principles, even when requested to report results for the same measurand, have proved challenging for PTs offering participant-derived consensus values.

The need for improvements has also been identified for the LC-MS methods. Due care is recommended to achieve proper equilibration after the addition of standards, a complete extraction and a complete digestion of proteins required for an equimolar release of peptides from the allergenic proteins.

The ENFADL will pursue its efforts in the harmonisation and validation of measurement procedures to achieve a reliable and consistent detection of allergens in food products among the EU Member States. The JRC envisages to develop a dedicated certified reference material that could 
be used as a common calibrant to allow comparable results from ELISA test kits and the LC-MS methods.

Open Access This article is licensed under a Creative Commons Attribution 4.0 International License, which permits use, sharing, adaptation, distribution and reproduction in any medium or format, as long as you give appropriate credit to the original author(s) and the source, provide a link to the Creative Commons licence, and indicate if changes were made. The images or other third party material in this article are included in the article's Creative Commons licence, unless indicated otherwise in a credit line to the material. If material is not included in the article's Creative Commons licence and your intended use is not permitted by statutory regulation or exceeds the permitted use, you will need to obtain permission directly from the copyright holder. To view a copy of this licence, visit http://creativecommons.org/licenses/by/4.0/.

\section{References}

1. Commission Regulation (EU) No 1169/2011 of the European Parliament and of the Council on the provision of food information to consumers, Official Journal L 304. http://data.europa.eu/eli/reg/ 2011/1169/oj. Accessed 01 Dec 2020

2. Regulation (EU) No 178/2002 of the European Parliament and of the Council of 28 January 2002 laying down the general principles and requirements of food law, establishing the European Food Safety Authority and laying down procedures in matters of food safety, Official Journal L 31. http://data.europa.eu/eli/reg/2002/ 178/oj. Accessed 01 Dec 2020

3. O'Connor GHM, Ulberth F (2017) Joint DG SANTE and DG JRC workshop: harmonisation of approaches for informing EU allergen labelling legislation. Rep JRC108259. https://bit.ly/2xnNl 4i. Accessed 01 Dec 2020

4. Matricardi PM, Kleine-Tebbe J, Hoffmann HJ, Valenta R, Hilger C, Hofmaier S et al (2016) EAACI molecular allergology user's guide. Pediatric Allergy Immunol 27(23):1-250. https://doi.org/ 10.1111/pai.12563
5. European Union RASFF-Rapid Alert System for Food and Feed portal. https://ec.europa.eu/food/safety/rasff_en. Accessed 01 Dec 2020

6. Martinez-Esteso MJ, O'Connor G, Nørgaard J, Breidbach A, Brohée M, Cubero-Leon E, Nitride C, Robouch P, Emons H (2020) A reference method for determining the total allergenic protein content in a processed food: the case of milk in cookies as proof of concept. Anal Bioanal Chem 412:8249-8267. https:// doi.org/10.1007/s00216-020-02959-0

7. ISO Guide 35:2017 Reference materials: guidance for characterization and assessment of homogeneity and stability. International Organization for Standardization, Geneva, Switzerland

8. ISO 13528:2015 Statistical methods for use in proficiency testing by interlaboratory comparisons. International Organization for Standardization, Geneva, Switzerland

9. SoftCRM, http://www.eie.gr/iopc/softcrm/index.html. Accessed 03 Aug 2020

10. Muñoz A, Kral R, Schimmel H (2011) Quantification of protein calibrants by amino acid analysis using isotope dilution mass spectrometry. Anal Biochem 408:124-31. https://www.sciencedir ect.com/science/article/pii/S0003269710005658

11. Gellrich K, Meyer HHD, Wiedemann S (2014) Composition of major proteins in cow milk differing in mean protein concentration during the first 155 days of lactation and the influence of season as well as short term restricted feeding in early and mid-lactation. Czech J Anim Sci 59: 97-106. https://www.agriculturejournals. cz/publicFiles/116521.pdf

12. Nitride C, Nørgaard J, Omar J, Emons H, Esteso MJ, O'Connor G (2019) An assessment of the impact of extraction and digestion protocols on multiplexed targeted protein quantification by mass spectrometry for egg and milk allergens. Anal Bioanal Chem 411:3463-3475. https://doi.org/10.1007/s00216-019-01816-z

Publisher's Note Springer Nature remains neutral with regard to jurisdictional claims in published maps and institutional affiliations. 